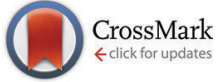

Cite this: New J. Chem., 2016, 40, 1655

Received (in Montpellier, France) 7th September 2015 Accepted 8th December 2015 DOI: $10.1039 / c 5 n j 02394 g$

www.rsc.org/njc

\title{
Effect of fluorination pattern and extent on the properties of PCDTBT derivatives $\dagger$
}

\author{
Luke Cartwright, Hunan Yi and Ahmed Iraqi*
}

\begin{abstract}
Herein, we report the synthesis of a series of fluorinated dithienyl carbazole-alt-benzothiadiazoles (PCDTBT analogues) and the characterisation of their optical, electrochemical, thermal and molecular organisation in the solid state. The polymers were decorated with fluorine on either the benzothiadiazole unit, carbazole unit or both to yield PCDTffBT, PCffDTBT and PCffDTffBT, respectively. The copolymers displayed decomposition temperatures in excess of $350{ }^{\circ} \mathrm{C}$. PCDTffBT, PCffDTBT and PCffDTffBT displayed optical band gaps of $1.86,1.82$ and $1.88 \mathrm{eV}$, respectively. It was speculated this was a consequence of the higher molecular weight of PCffDTBT relative to the other polymers. PCffDTBT and PCffDTffBT displayed shallower HOMO levels relative to PCDTffBT; a consequence of fluorinating the carbazole-donor moiety. XRD studies confirmed that fluorinating the benzothiadiazoleacceptor moiety improves molecular ordering by promoting $\pi-\pi$ stacking of polymer backbones in solid state. Interestingly, fluorinating the carbazole-donor unit does not improve $\pi-\pi$ stacking of polymer backbones.
\end{abstract}

\section{Introduction}

Carbazole, is an appealing building-block for the development of conjugated copolymers. ${ }^{1}$ Carbazole is structurally analogous to fluorene, which has also found widespread use in optoelectronic devices. However, the presence of an electron-donating nitrogen atom in its central fused pyrrole ring renders carbazole fully aromatic, unlike fluorene. ${ }^{1}$ Replacing the $9 H$-carbon with nitrogen in the fluorene skeleton bestows further desirable properties upon the resulting unit including: (1) increased oxidative stability owing to the propensity for the $9 H$-position of fluorene to undergo oxidation to the ketone product, which results in adverse physical properties. $^{2}$ (2) The nitrogen in the central pyrrole ring can be functionalised to improve the physical properties of the resulting polymer. ${ }^{3-5}$ Consequently, carbazole-based conjugated polymers are easy to synthesise, display excellent photochemical and thermal stabilities and possess high charge carrier mobilities. ${ }^{1}$ Thus, carbazole-based conjugated polymers and their derivatives have found extensive use in organic light emitting diodes (OLEDs), organic thermal electronic devices, organic field-effect transistors (OFETs) and organic photovoltaics (OPV). ${ }^{6-11}$

It could be argued that poly[ $N$-9'-heptadecanyl-2,7-carbazolealt-5,5-(4',7'-di-2-thienyl-2', $1^{\prime}, 3^{\prime}$-benzothiadiazole)] (PCDTBT) and

Department of Chemistry, University of Sheffield, Sheffield, S3 7HF, UK.

E-mail: a.iraqi@sheffield.ac.uk.ac.uk; Fax: +44(0)114 222 9303;

Tel: +44 (0)1142229566

$\dagger$ Electronic supplementary information (ESI) available: ${ }^{1} \mathrm{H}-\mathrm{NMR}$ spectra of the final monomers and the polymers. See DOI: $10.1039 / \mathrm{c} 5 \mathrm{nj} 02394 \mathrm{~g}$ its derivatives are the most studied carbazole-based donoracceptor $(\mathrm{D}-\pi-\mathrm{A})$ conjugated polymers. PCDTBT has found widespread use in organic photovoltaic (OPV) devices and efficiencies in excess of $7 \%$ have been achieved when blended with the fullerene derivative $\mathrm{PC}_{70} \mathrm{BM} .{ }^{10}$ This is by no means the highest efficiency recorded in OPV devices. ${ }^{12-14}$ However, the thermal and oxidative stability, deep HOMO level and ease of processability have allowed PCDTBT to remain an attractive conjugated polymer for optoelectronic applications. ${ }^{15,16}$

The body of literature concerning the incorporation of fluorine as a substituent and the effect it has on the resulting polymer system is expanding rapidly. ${ }^{17-20}$ However, fluorination of PCDTBT has received little attention, which is surprising considering the promise PCDTBT and its derivatives have displayed in optoelectronic devices. ${ }^{21-24}$ Imahori and co-workers investigated the effects of fluorine substitution on PCDTBT by copolymerising a fluorinated benzothiadiazole-acceptor with a carbazole-donor to yield PCDTBT-F. ${ }^{24}$ The introduction of two fluorine atoms on the 5,6-positions of benzothiadiazole decreased the HOMO energy level of PCDTBT-F, relative to that of PCDTBT. However, PCDTBT-F displayed a significantly reduced molecular weight, poor solubility, blue-shifted absorption maxima and a larger optical band-gap, relative to PCDTBT. $^{24}$ In contrast, Bo et al. reported the polymerisation of a fluorinated-carbazole donor with a dialkoxy-substituted benzothiadiazole acceptor to yield PDFCDTBT. ${ }^{21}$ The introduction of fluorine atoms at the 3,6-positions of carbazole yielded a moderate molecular weight polymer with deeper frontier energy levels, red-shifted absorption maxima and a smaller 


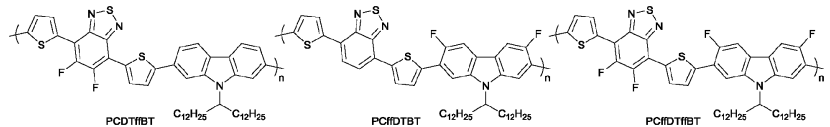

Fig. 1 Structures of PCDTffBT, PCffDTBT and PCffDTffBT

optical band gap, relative to its non-fluorinated counterpart. Furthermore, PDFCDTBT displayed more organised packing in solid state. ${ }^{21}$

With this is mind, we report the synthesis of three fluorinated carbazole-alt-benzothiadiazole conjugated polymers. Fluorine was attached to either the benzothiadiazole unit, carbazole unit or both units to yield PCDTffBT, PCffDTBT and PCffDTffBT, respectively (Fig. 1). Imahori reported that the fluorinated PCDTBT analogue they synthesised forms a low molecular weight polymer with poor solubility. Thus, the polymers in this work were designed to bear larger solubilising chains on the carbazole moiety in the hope that higher molecular weight polymers could be obtained. The optical, electrochemical, thermal and structural properties of the polymers in the solid state are probed in order to ascertain the effects fluorination has on the resulting polymer.

\section{Results and discussion}

\section{Polymer synthesis}

The synthetic route for the preparation of 2,7-dibromo-3,6-difluoro9-(pentacosan-13-yl)-9H-carbazole (M1) is outlined in Scheme 1. M1 was synthesised starting from the commercially available 1,4-dibromo-2-fluorobenzene. 2,7-Dibromo-9-(pentacosan-13yl)-9H-carbazole (M2) was synthesised according to a modified literature procedure. ${ }^{25}$ 4,7-Bis(5-(trimethylstannyl)thiophene-2yl)benzo[c][1,2,5]thiadiazole (M3) and 5,6-difluoro-4,7-bis(5-(trimethylstannyl)thiophene-2-yl)benzo[c][1,2,5]thiadiazole (M4) were synthesised from 4,7-di(thiophen-2-yl)benzo[c] $][1,2,5]$ thiadiazole and 5,6-difluoro-4,7-di(thiophene-2-yl)benzo[c][1,2,5]thiadiazole, respectively. Deprotonation of the starting material with lithium 2,2,6,6-tetramethylpiperidine (LTMP) and subsequent treatment with trimethyltin chloride afforded M3 and M4 (Scheme 1). Stille polycondensation of M2 with M4, M1 with M3 and M1 with M4 yielded PCDTffBT, PCffDTBT and PCffDTffBT, respectively (Scheme 1). $\mathrm{Pd}(\mathrm{OAc})_{2}$ and tri $\left(o\right.$-tolyl) phosphine $\left(\mathrm{P}(o \text {-tol })_{3}\right)$ were used

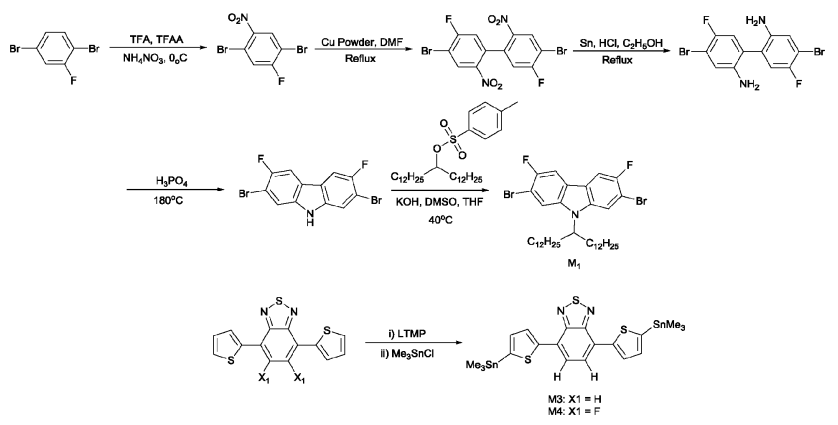

Scheme 1 Synthetic routes to the final monomer products: M1, M3 and M4. M2 was synthesised using previously established literature procedures. as the catalyst and toluene as the solvent. All polymerisations were left for 48 hours. The polymers were cleaned via Soxhlet extraction using in turn methanol, acetone, hexane and toluene. The toluene fractions were collected, reduced in vacuo and precipitated in methanol. Subsequent studies were conducted on the toluene fractions only. ${ }^{1} \mathrm{H}$-NMR and elemental analysis were used to verify the structure of PCDTffBT, PCffDTBT and PCffDTffBT. Gel permeation chromatography (GPC) using 1,2,4-trichlorobenzene as the eluent, at $140{ }^{\circ} \mathrm{C}$ was used to determine the number-average molecular weight $\left(M_{\mathrm{n}}\right)$ and weight-average molecular weight $\left(M_{\mathrm{w}}\right)$ of the polymers synthesised. PCffDTBT displayed the highest $M_{\mathrm{n}}$ and $M_{\mathrm{w}}$ of all polymers synthesised with values of 11400 and 20600 Da, respectively. Both PCDTffBT and PCffDTffBT displayed significantly reduced molecular weights relative to PCffDTBT with values of 11900 and $8900 \mathrm{Da}$, respectively (Table 1). Previous literature has speculated that fluorination of the benzothiadiazole unit moiety promotes $\pi-\pi$ stacking and aggregation of polymer chains, which impedes the solubility of the polymer and its attainable molecular weight. ${ }^{26}$ The results obtained here support this hypothesis.

PCDTBT-F synthesised by Imahori and co-workers is an analogous polymer to PCDTffBT. ${ }^{24}$ PCDTBT-F has C17branched substituents rather than C25-branched substituents for PCDTffBT. It was reported that PCDTBT-F displayed poor solubility which limited the molecular weight of the resulting polymer $\left(M_{\mathrm{w}}=6000 \mathrm{Da}\right)$. Thus, PCDTffBT was designed to bear larger solubilising chains on the carbazole moiety in the hope that a higher molecular weight polymer could be obtained. PCDTffBT does possess a higher $M_{\mathrm{w}}(11900 \mathrm{Da})$, relative to PCDTBT-F. PCffDTffBT in which both the carbazole and benzothiadiazole repeat units have fluorine substituents, has the most reduced processability in this series of polymers. Both the yield of the soluble toluene fraction and the molecular weight of this polymer are lower compared to the other two polymers presented in this work.

\section{Optical properties}

The optical properties of all polymers were investigated by UV-vis absorption spectroscopy on dilute chloroform solutions and drop-cast films on quartz substrates (Fig. 2). The optical properties of the polymers are summarised in Table 1 . All polymers exhibit two absorption peaks in both solution and film states. The absorption band at shorter wavelengths can be ascribed to the $\pi-\pi^{*}$ transition. In contrast, the absorption band at longer wavelengths can be attributed to intramolecular charge transfer between the dithienyl-carbazole donor moieties and the benzothiadiazole acceptor units. All polymers undergo a bathochromic shift when cast into a thin-film, relative to their solution absorption. This can be ascribed to stronger $\pi-\pi$ interchain stacking and a more coplanar structure in solid state. The $\lambda_{\max }$ of PCDTffBT, PCffDTBT and PCffDTffBT are located at 556, 558 and $545 \mathrm{~nm}$ in film states. The optical band gaps of PCDTffBT, PCffDTBT and PCffDTffBT, as determined from the onset of absorption, were estimated to be 1.86, 1.82 and $1.88 \mathrm{eV}$, respectively. Furthermore, PCffDTffBT displayed improved resolution in the solid-state with the presence 
Table 1 A summary of the GPC, UV-vis absorption and fluorescence data for PCDTffBT, PCffDTBT and PCffDTffBT

\begin{tabular}{|c|c|c|c|c|c|c|c|c|c|c|}
\hline \multirow[b]{2}{*}{ Polymer } & \multirow[b]{2}{*}{$M_{\mathrm{n}}{ }^{a}$} & \multirow[b]{2}{*}{$M_{\mathrm{w}}{ }^{a}$} & \multirow[b]{2}{*}{ PDI } & \multicolumn{4}{|c|}{ UV-vis absorption } & \multicolumn{3}{|l|}{ Fluorescence } \\
\hline & & & & $\begin{array}{l}\lambda_{\max } \\
\text { solution }(\mathrm{nm})\end{array}$ & $\begin{array}{l}\varepsilon^{b} \\
\left(\mathrm{M}^{-1} \mathrm{~cm}^{-1}\right)\end{array}$ & $\begin{array}{l}\lambda_{\max } \\
\text { film }(\mathrm{nm})\end{array}$ & $\begin{array}{l}E_{\mathrm{g}}^{\mathrm{opt}} \\
\text { film }^{c}(\mathrm{eV})\end{array}$ & $\begin{array}{l}\lambda_{\max } \\
\text { solution }(\mathrm{nm})\end{array}$ & $\begin{array}{l}\lambda_{\max } \\
\text { film }(\mathrm{nm})\end{array}$ & $\begin{array}{l}\text { Stokes shift } \\
(\mathrm{nm})\end{array}$ \\
\hline PCDTffBT & 7700 & 11900 & 1.55 & 379,526 & 49900 & 395,556 & 1.86 & 618 & 710 & 154 \\
\hline PCffDTBT & 11400 & 20600 & 1.81 & 381,526 & 40600 & 404,558 & 1.82 & 637 & 712 & 154 \\
\hline PCffDTffBT & 6100 & 8900 & 1.46 & 387,520 & 43000 & 401,545 & 1.88 & 600 & 712 & 167 \\
\hline
\end{tabular}

${ }^{a}$ Measurements conducted on the toluene fraction of the polymer using differential refractive index (DRI) detection. ${ }^{b}$ Absorption coefficient measured at $\lambda_{\max }$ in chloroform solution. ${ }^{c}$ Optical energy gap determined from the onset of the absorption band in thin film. ${ }^{d}$ Stokes shift determined from film studies.
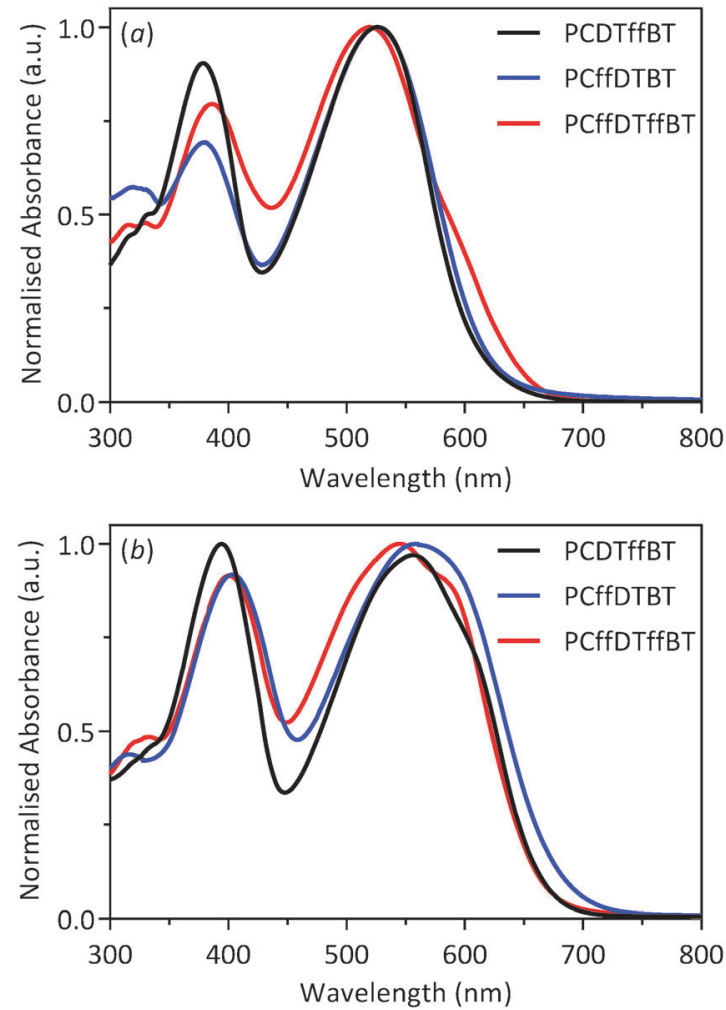

Fig. 2 Normalised UV-vis absorption spectra of PCDTffBT, PCffDTBT and PCffDTffBT in: (a) chloroform solution; and (b) thin films.

of shoulder peak at $\sim 580 \mathrm{~nm}$, which we attribute to a higher tendency of aggregation of polymer chains for this polymer compared to the other two polymers in the series; a consequence of improved molecular arrangement in solid state. The higher $M_{\mathrm{w}}$ of PCffDTBT did not translate into significantly higher $\lambda_{\max }$ value. However, the optical band gap of PCffDTBT was lower than PCDTffBT and PCffDTffBT. It would appear that in this series of polymers, fluorination of the carbazole moiety affords a polymer with the lowest optical band gap allowing the resulting polymer to harvest a larger portion of the solar spectrum. It is also worth noting that the optical band gap of non-fluorinated PCDTBT $(1.88 \mathrm{eV})^{\mathbf{1 1}}$ is similar to those of PCDTffBT and PCffDTffBT.

The fluorescence spectra of the polymers in solution and solid films are illustrated in Fig. 3. All fluorescence spectra were recorded upon exciting the polymers at their absorption
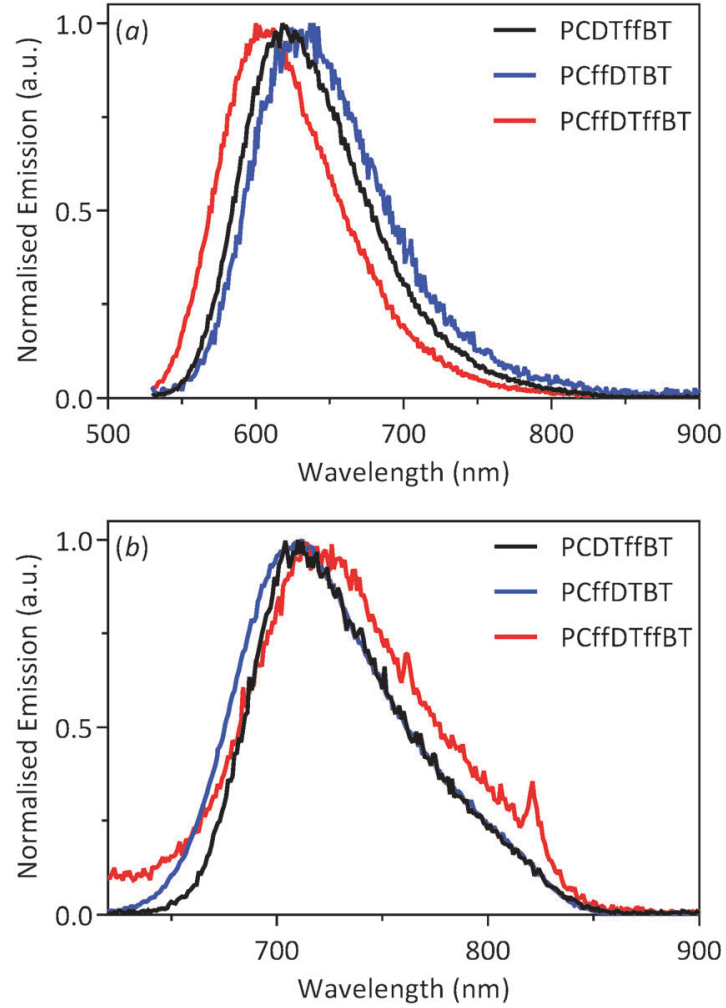

Fig. 3 Normalised fluorescence spectra of PCDTffBT, PCffDTBT and PCffDTffBT in: (a) chloroform solution; and (b) thin films.

maxima. In solution PCDTffBT, PCffDTBT and PCffDTffBT exhibited emission maxima at 618, 637 and $600 \mathrm{~nm}$, respectively. The emission maxima are red-shifted to 710,712 and $712 \mathrm{~nm}$ for PCDTffBT, PCffDTBT and PCffDTffBT, respectively. It is suspected that the large difference in the solution emission maxima is due to interactions between the local solvent environment and the fluorophore. Solvent molecules stabilise and lower the energy of the excited state of PCffDTBT; resulting in a red-shift in the solution fluorescence emission. Once the polymers have been cast into films, the effects of solvent relaxation no longer apply, and the polymers exhibit a fluorescence emission at similar wavelengths. The large Stokes shifts of 154, 154 and $167 \mathrm{~nm}$ for PCDTffBT, PCffDTBT and PCffDTffBT, respectively, suggests there is a significant energy difference between the ground state and excited state of the polymers. 


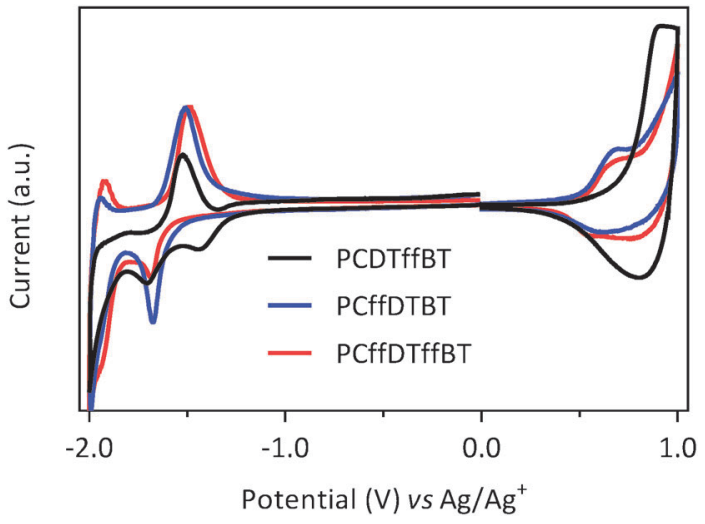

Fig. 4 Cyclic voltammograms of PCDTffBT, PCffDTBT and PCffDTffBT on platinum disc electrodes (area $0.031 \mathrm{~cm}^{2}$ ) at a scan rate of $100 \mathrm{mV} \mathrm{s}^{-1}$ in acetonitrile/tetrabutyl ammonium perchlorate $\left(0.1 \mathrm{~mol} \mathrm{dm}^{-3}\right)$.

\section{Electrochemical properties}

Cyclic voltammetry was used to determine the HOMO and LUMO energy levels ( $v s$. vacuum) of drop-cast polymer films in acetonitrile with tetrabutylammonium perchlorate as the electrolyte (Fig. 4 and Table 2). PCDTffBT displayed the deepest HOMO level of all polymers synthesised with a value of $-5.44 \mathrm{eV}$, which is deeper than its non-fluorinated analogue, PCDTBT. ${ }^{11}$ The LUMO level of PCDTffBT was measured to be $-3.45 \mathrm{eV}$, which is slightly lower than PCDTBT. ${ }^{11}$ This is consistent with previous literature concerning the effects of fluorine substitution and the effects it has the resulting polymers HOMO levels. ${ }^{27-31}$

The HOMO level of PCffDTBT was calculated to be $-5.23 \mathrm{eV}$. This is similar to the HOMO of level of PDFCDTBT reported by Bo and co-workers. ${ }^{21}$ However, it is significantly shallower than PCDTBT. ${ }^{11}$ The electrochemistry results suggest that fluorination of the carbazole-moiety results in it becoming a significantly stronger donor unit. Generally, fluorine is considered to be a deactivating substituent, thus, this result may seem erroneous. However, it is known that fluorine as a substituent on an aromatic benzene is considered to be both $\sigma$-electron withdrawing and $\pi$-electron donating. ${ }^{32}$ The $\pi$-donating ability of fluorine is reflected in the anomalously high para-position reactivity. ${ }^{33}$ Fluorobenzene displays a higher proton affinity than benzene, another example of the anomalously high reactivity of fluorobenzene relative to benzene. ${ }^{34,35}$ We speculate that fluorination of the carbazole moiety increases the $\pi$-electron density of the

Table 2 A summary of the TGA and electrochemical data for PCDTffBT, PCffDTBT and PCffDTffBT

\begin{tabular}{lllll}
\hline Polymer & $T_{\mathrm{d}}{ }^{a}\left({ }^{\circ} \mathrm{C}\right)$ & $\mathrm{HOMO}^{b}(\mathrm{eV})$ & $\operatorname{LUMO}^{c}(\mathrm{eV})$ & $E_{\mathrm{g}}^{\text {elec } d}(\mathrm{eV})$ \\
\hline PCDTffBT & 367 & -5.44 & -3.45 & 1.99 \\
PCffDTBT & 390 & -5.23 & -3.12 & 2.11 \\
PCffDTffBT & 351 & -5.24 & -3.11 & 2.13
\end{tabular}

${ }^{a}$ Onset of degradation as determined via thermogravimetric analysis (TGA) with a heating rate of $10{ }^{\circ} \mathrm{C} \mathrm{min}-1$ under an inert atmosphere of nitrogen. ${ }^{b}$ HOMO position (vs. vacuum) determined from the onset of oxidation. ${ }^{c}$ LUMO position (vs. vacuum) determined from the onset of reduction. ${ }^{d}$ Electrochemical energy gap. resulting monomer unit. Consequently, the fluorinated carbazole moiety is a stronger donor resulting in a shallower HOMO level. It is possible that this will negatively impact the oxidative stability of the PCffDTBT.

\section{Thermal properties}

Thermogravimetric analysis of PCDTffBT, PCffDTBT and PCffDTffBT revealed that all polymers possess good thermal stability with degradation temperatures in excess of $350{ }^{\circ} \mathrm{C}$ (Fig. 5). The onset of degradation (5\% weight loss) for PCDTffBT, PCffDTBT and PCffDTfBT were estimated to be 367,390 and $351{ }^{\circ} \mathrm{C}$ (Table 2). We tentatively speculate that the degradation temperature of these polymers is dependent upon the molecular weight. We hypothesise that the lower molecular weights of PCDTffBT and PCffDTffBT have two effects on the polymer properties. Firstly, PCDTffBT and PCffDTffBT possess a higher concentration of polymer chain ends, which can undergo chain-end scission. Secondly, degradation mechanisms involve molecular mobility which is inversely related to molecular weight. An increased molecular weight should reduce the amount of labile end-groups facilitating chain entanglement. Thus, the molecular mobility of the polymer, which controls the kinetics of decomposition, is reduced.

\section{X-Ray diffraction (XRD) studies}

Powder X-ray diffraction (XRD) patterns of polymers PCDTffBT, PCffDTBT and PCffDTffBT were obtained to investigate the molecular organisation of polymers in the solid state (Fig. 6). All polymers display peaks in the low angle region, which suggests the polymers possess some long-range translational order. An indication the polymers adopt a more crystalline structure in the solid state. The low-angle peak in the XRD pattern is frequently observed in $\pi$-conjugated polymers with long alkyl or alkoxy side-chains. ${ }^{36-38}$ Previous literature has shown that this peak is attributed to the distance between $\pi$-conjugated backbones which are separated by the solubilising alkyl chains. ${ }^{39,40}$

Interestingly, PCDTffBT and PCffDTffBT display additional wide angle peaks at $2 \theta=25.6^{\circ}$, which corresponds to a

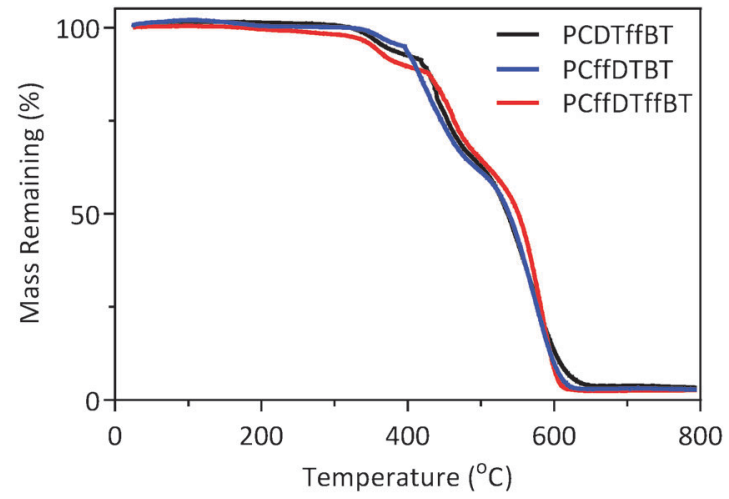

Fig. 5 Thermogravimetric analysis of PCDTffBT, PCffDTBT and PCffDTffBT with a heating rate of $10{ }^{\circ} \mathrm{C} \mathrm{min}^{-1}$ under an inert nitrogen atmosphere. 


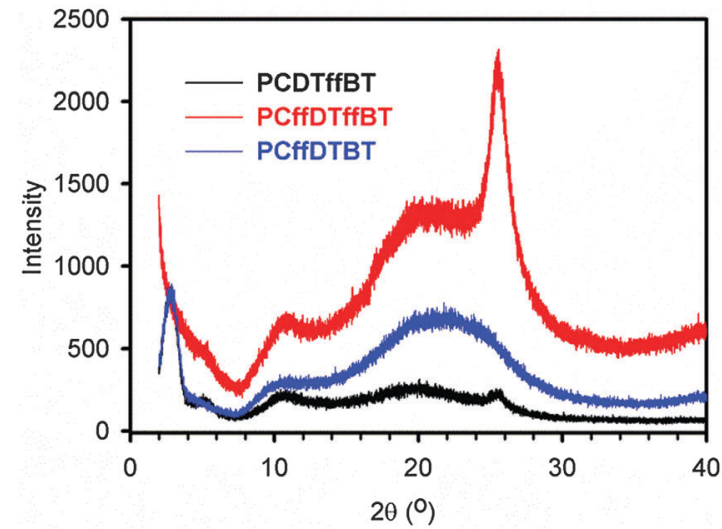

Fig. 6 Powder X-ray diffraction (XRD) patterns of PCDTffBT, PCffDTBT and PCffDTffBT.

$\pi-\pi$ stacking distance of $3.48 \AA$. It is speculated that fluorinating the benzothiadiazole acceptor unit improves molecular ordering by promoting interactions with components on adjacent aromatics. This improves the planarity of the resulting backbone, resulting in strong interchain interactions and a more pronounced packing order. A result consistent with the reduced inhomogeneous broadening observed in the solid state spectra of PCffDTffBT. PCffDTBT does not display an additional peak in this area suggesting that fluorination of the carbazole moiety does have the same impact. Interestingly, Bo et al. found that PDFCDTBT, an analogous polymer to PCffDTBT, displayed a $\pi-\pi$ stacking distance of $3.7 \AA^{21}$ However, the carbazole moiety in PDFCDTBT was substituted with a significantly smaller alkyl chain (C8 linear chain vs. C25 branched chain). It is hypothesised that the larger alkyl chain in PCffDTBT disrupts the formation of $\pi-\pi$ stacking in solid state.

\section{Conclusions}

In summary, three fluorinated carbazole-alt-benzothiadiazole D- $\pi-A$ alternating copolymers PCDTffBT, PCffDTBT and PCffDTffBT were synthesised via Stille polymerisation using toluene as the solvent. The optical, thermal, electrochemical and structural properties of these polymers were analysed and contrasted in relation to the degree of fluorination and their fluorination pattern. The optical band gaps of PCDTffBT, PCDTffBT and PCffDTffBT were estimated to be $1.86,1.82$ and $1.88 \mathrm{eV}$, respectively. It is hypothesised that the lower optical band gap of PCffDTBT was a consequence of its higher molecular weight. The HOMO levels of PCffDTBT $(-5.23 \mathrm{eV})$ and PCffDTffBT $(-5.24 \mathrm{eV})$ were significantly shallower relative to that of PCDTfBBT $(-5.44 \mathrm{eV})$. It is hypothesised that the introduction of fluorine at the 3,6-positions of the carbazole unit increases its donating ability resulting in a shallower HOMO level. Thermogravimetric analysis revealed that the onset of decomposition is strongly dependent upon the molecular weight of the resulting polymer with PCffDTBT and PCffDTffBT displaying the highest and lowest decomposition temperatures, respectively. Powder X-ray diffraction revealed that all polymers possess peaks in the low angle region suggesting all polymers have some long range translational order. PCDTffBT and PCffDTfBT both displayed sharp peaks at $2 \theta$ values of $25.6^{\circ}$. These peaks are indicative of $\pi-\pi$ stacking suggesting fluorination of the benzothiadiazole moiety improves the planarity of the polymer backbone, leading to a more pronounced packing order in the solid state. PCffDTBT did not display a shark peak at this angle.

\section{Experimental}

\section{Experimental}

All materials were purchased from commercial suppliers and used as received, unless otherwise stated. Toluene was dried and distilled over sodium under an inert argon atmosphere. Acetonitrile was dried and distilled over phosphorous pentoxide under an inert argon atmosphere, then stored over molecular sieves $(3 \AA)$.

\section{Measurements}

${ }^{1} \mathrm{H}$ and ${ }^{13} \mathrm{C}$ nuclear magnetic resonance (NMR) spectra were recorded on a Bruker AV $400(400 \mathrm{MHz})$ using chloroform-d $\left(\mathrm{CDCl}_{3}\right)$ or acetone-d as the solvent. ${ }^{1} \mathrm{H}-\mathrm{NMR}$ of the polymers were recorded on Bruker Avance III HD 500 (500 MHz) spectrometer at $100{ }^{\circ} \mathrm{C}$ using 1,2-dideutrotetrachloroethance as the solvent. Coupling constants are given in Hertz $(\mathrm{Hz})$. Carbon, hydrogen, nitrogen and sulphur elemental analysis was performed on a Perkin Elmer 2400 series 11 CHNS/O analyser. Analysis of halides was undertaken using the Schöniger flask combustion method. GPC analysis was conducted on polymer solutions using 1,2,4-trichlorobenzene at $140{ }^{\circ} \mathrm{C}$ as the eluent. Polymer samples were spiked with toluene as a reference. GPC curves were obtained using a Viscotek GPCmax VE2001 GPC solvent/sample module and a Waters 410 Differential Refractometer, which was calibrated using a series of narrow polystyrene standards (Polymer Laboratories). TGA's were obtained using a Perkin Elmer TGA-1 Thermogravimetric Analyser at a scan rate of $10{ }^{\circ} \mathrm{C} \mathrm{min}^{-1}$ under an inert nitrogen atmosphere. Powder X-ray diffraction samples were recorded on a Bruker D8 advance diffractometer with a CuK $\alpha$ radiation source $(1.5418 \AA$, rated as $1.6 \mathrm{~kW}$ ). The scanning angle was conducted over the range $2-40^{\circ}$. UV-visible absorption spectra were recorded using a Hitachi U-2010 Double Bean UV/Visible Spectrophotometer. Polymer solutions were made using chloroform and measured using quartz cuvettes (path length $=1 \times 10^{-2} \mathrm{~m}$ ). Thin films, used for absorption spectra were prepared by drop-casting solutions onto quartz plates using $1 \mathrm{mg} \mathrm{cm}^{-3}$ polymer solutions that were prepared with chloroform. Fluorescence spectra were recorded on a Horiba FluoroMax 4 spectrometer. Polymer solutions were made using chloroform and measured using quartz cuvettes (path length $=1 \times 10^{-2} \mathrm{~m}$ ). Thin films, were prepared by drop-casting solutions onto quartz plates using $5 \mathrm{mg} \mathrm{cm}^{-3}$ polymer solutions that were prepared with chlorobenzene. Cyclic voltammograms were recorded using a Princeton Applied Research Model 263A Potentiostat/Galvanostat. 
A three electrode system was employed comprising a Pt disc, platinum wire and $\mathrm{Ag} / \mathrm{Ag}^{+}$as the working electrode, counter electrode and reference electrode, respectively. Measurements were conducted in a tetrabutylammonium perchlorate acetonitrile solution $\left(0.1 \mathrm{~mol} \mathrm{dm}^{-3}\right)$ on polymer films that were prepared by drop casting polymer solution. Ferrocene was employed as the reference redox system; in accordance with IUPAC's recommendations. ${ }^{41}$ The energy level of $\mathrm{Fc} / \mathrm{Fc}^{+}$was assumed at $-4.8 \mathrm{eV}$ to vacuum. The half-wave potential of $\mathrm{Fc} / \mathrm{Fc}^{+}$redox couple was found to be $0.08 \mathrm{~V} v s$. $\mathrm{Ag} / \mathrm{Ag}^{+}$reference electrode. The HOMO energy levels of polymers were estimated by equation: $E_{\mathrm{HOMO}}=-\left(4.8-E_{1 / 2, \mathrm{Fc}, \mathrm{Fc}^{+}}+E_{\text {ox, onset }}\right)=-(4.72+$ $\left.E_{\text {ox,onset }}\right) \mathrm{eV}$, where $E_{\text {ox,onset }}$ is the onset oxidation potential relative to the $\mathrm{Ag} / \mathrm{Ag}^{+}$reference electrode. The LUMO energy levels of polymers were calculated using the equation: $E_{\mathrm{LUMO}}=$ $-\left(4.8-E_{1 / 2, \mathrm{Fc}, \mathrm{Fc}^{+}}+E_{\text {red,onset }}\right)=-\left(4.72+E_{\text {red,onset }}\right) \mathrm{eV}$, where $E_{\text {red,onset }}$ is the onset reduction potential relative to the $\mathrm{Ag} / \mathrm{Ag}^{+}$ reference electrode.

3,6-Difluoro-2,7-dibromo-9-(pentacosan-13-yl)-9H-carbazole (M1). 2,7-Dibromo-3,6-difluoro-9H-carbazole ${ }^{42}(3.0 \mathrm{~g}, 15.4 \mathrm{mmol})$ and powdered $\mathrm{KOH}(4.31 \mathrm{~g}, 41.8 \mathrm{mmol})$ were dissolved in anhydrous DMSO $\left(100 \mathrm{~cm}^{3}\right)$. The mixture was heated to $40{ }^{\circ} \mathrm{C}$ and pentacosan-13-yl 4-methylbenzene (10.41 g, $19.9 \mathrm{mmol})$ in dry THF $\left(10 \mathrm{~cm}^{3}\right)$ was added dropwise over 2 hours. The reaction was allowed to stir overnight. Upon completion, the reaction was poured on $\mathrm{H}_{2} \mathrm{O}\left(300 \mathrm{~cm}^{3}\right)$ and the product extracted with hexane $\left(4 \times 300 \mathrm{~cm}^{3}\right)$. The organic fractions were combined, dried $\left(\mathrm{MgSO}_{4}\right)$ and the solvent removed in vacuo. The product was purified via silica gel column chromatography using hexane as the eluent to yield an off-white solid (2.43 g, $3.41 \mathrm{mmol}, 22 \%)$. ${ }^{1} \mathrm{H}$ NMR (400 MHz, $\mathrm{CDCl}_{3}$ ) $\delta$ (ppm): 7.74 (br, 3H), $7.58(\mathrm{br}, 1 \mathrm{H})$, 4.39 (sep, $J=5.09 \mathrm{~Hz}, 1 \mathrm{H}), 2.19$ (m, 2H), 1.92 (m, 2H), 1.36-1.08 $(\mathrm{m}, 38 \mathrm{H}), 0.97(\mathrm{~m}, 2 \mathrm{H}), 0.89(\mathrm{t}, J=6.94 \mathrm{~Hz}, 6 \mathrm{H}) .{ }^{13} \mathrm{C} \mathrm{NMR}$ $\left(250 \mathrm{MHz}, \mathrm{CDCl}_{3}\right) \delta$ (ppm): 154.29, 151.92, 139.54, 135.89, 115.62, 113.40, 106.88, 57.35, 33.57, 31.75, 29.49, 29.37, 29.28, 29.12, 26.73, 22.62, 14.07. ${ }^{19} \mathrm{~F}$ NMR (250 MHz, $\left.\mathrm{CDCl}_{3}\right) \delta(\mathrm{ppm})$ : -118.54, -119.06. EI-MS (m/z): $[\mathrm{M}]^{+}$calculated for $\mathrm{C}_{37} \mathrm{H}_{55} \mathrm{Br}_{2} \mathrm{~F}_{2} \mathrm{~N}$, 711.2710; found, 711.2685. Anal. calculated for $\mathrm{C}_{37} \mathrm{H}_{55} \mathrm{Br}_{2} \mathrm{~F}_{2} \mathrm{~N}$ : C, 62.45; H, 7.71; N, 1.97; Br, 22.46; found: C, 62.08; H, 7.92; N, 1.95; $\mathrm{Br}, 23.22$.

2,7-Dibromo-9-(pentacosan-13-yl)-9H-carbazole (M2). 2,7Dibromo-9H-carbazole $(5.00 \mathrm{~g}, 15.4 \mathrm{mmol})$ and powdered $\mathrm{KOH}$ (4.31 g, $76.9 \mathrm{mmol}$ ) were dissolved in anhydrous DMSO $\left(100 \mathrm{~cm}^{3}\right)$. The mixture was heated to $40{ }^{\circ} \mathrm{C}$ and pentacosan-13yl 4-methylbenzene $(12.06 \mathrm{~g}, 23.08 \mathrm{mmol})$ in dry THF $\left(20 \mathrm{~cm}^{3}\right)$ was added dropwise over 2 hours. The reaction was allowed to stir overnight. Upon completion, the reaction was poured on $\mathrm{H}_{2} \mathrm{O}\left(300 \mathrm{~cm}^{3}\right)$ and the product extracted with hexane $\left(4 \times 300 \mathrm{~cm}^{3}\right)$. The organic fractions were combined, dried $\left(\mathrm{MgSO}_{4}\right)$ and the solvent removed in vacuo. The product was purified via silica gel column chromatography using hexane as the eluent to yield an off-white solid (4.83 g, $7.15 \mathrm{mmol}, 46 \%){ }^{1} \mathrm{H}$ NMR (400 MHz, $\left.\mathrm{CDCl}_{3}\right) \delta(\mathrm{ppm}): 7.92(\mathrm{dd}, J=4.51$ and $J=8.42 \mathrm{~Hz}, 2 \mathrm{H}), 7.73$ $(\mathrm{s}, 1 \mathrm{H}), 7.57(\mathrm{~s}, 1 \mathrm{H}), 7.35(\mathrm{t}, J=7.11 \mathrm{~Hz}, 2 \mathrm{H}), 4.44(\mathrm{sep}, J=2.51 \mathrm{~Hz}$, $1 \mathrm{H}), 2.21(\mathrm{~m}, 2 \mathrm{H}), 1.93(\mathrm{~m}, 2 \mathrm{H}), 1.36-1.09(\mathrm{~m}, 38 \mathrm{H}), 0.99(\mathrm{~m}, 2 \mathrm{H})$, $0.90(\mathrm{t}, J=6.94 \mathrm{~Hz}, 6 \mathrm{H}) .{ }^{13} \mathrm{C} \mathrm{NMR}\left(250 \mathrm{MHz}, \mathrm{CDCl}_{3}\right) \delta(\mathrm{ppm})$ :
$122.34,121.47,121.22,114.54,112.17,56.98,33.51,31.94,29.63$, 29.56, 29.49, 29.37, 29.33, 26.76, 22.72, 14.16 EI-MS (m/z): [M] $]^{+}$ calculated for $\mathrm{C}_{39} \mathrm{H}_{57} \mathrm{Br}_{2} \mathrm{NNa}$, 696.2755; found, 696.2730. Anal. calculated for $\mathrm{C}_{37} \mathrm{H}_{57} \mathrm{Br}_{2} \mathrm{~N}$ : C, 65.72; H, 8.50; N, 2.07; Br, 23.65; found: C, 64.37; H, 8.39; N, 1.94; Br, 27.61.

4,7-Bis(5-(trimethylstannyl)thiophene-2-yl)benzo[c] $][1,2,5]$ thiadiazole (M3). Under an inert argon atmosphere, 2,2,6,6tetramethylpiperdine $\left(1.46 \mathrm{~cm}^{3}, 8.66 \mathrm{mmol}\right)$ was dissolved in anhydrous THF $\left(20 \mathrm{~cm}^{3}\right)$. The solution was cooled to $-78{ }^{\circ} \mathrm{C}$ and $n$-butyl lithium $\left(3.46 \mathrm{~cm}^{3}, 8.66 \mathrm{mmol}, 2.5 \mathrm{M}\right.$ solution in hexane) was added dropwise. The resulting solution was allowed to stir at $-78{ }^{\circ} \mathrm{C}$. The reaction was then warmed to room temperature and stirred for 10 minutes. The solution was then cooled to $-78{ }^{\circ} \mathrm{C}$ and 4,7-di(thiophen-2-yl)benzo[ $[c][1,2,5]$ thiadiazole $(1.0 \mathrm{~g}, 3.33 \mathrm{mmol})$ in THF $\left(15 \mathrm{~cm}^{3}\right)$ was added dropwise. Upon complete addition, the solution was stirred at $-78{ }^{\circ} \mathrm{C}$ for 1 hour. Trimethyltin chloride $\left(8.32 \mathrm{~cm}^{3}, 8.33 \mathrm{mmol}\right.$, 1.0 $\mathrm{M}$ solution in THF) was added dropwise at $-78{ }^{\circ} \mathrm{C}$. The reaction was warmed to room temperature and left to stir overnight. Upon completion, saturated brine was added to quench the reaction. The product was extracted with DCM $\left(3 \times 100 \mathrm{~cm}^{3}\right)$, washed with brine $\left(3 \times 100 \mathrm{~cm}^{3}\right)$, dried $\left(\mathrm{MgSO}_{4}\right)$ and the solvent removed in vacuo. The crude material was recrystallised from ethanol to afford (M3) as orange needlelike crystals (1.42 g, $2.27 \mathrm{mmol}, 68 \%) .{ }^{1} \mathrm{H}$ NMR (400 MHz, $\left.\mathrm{CDCl}_{3}\right) \delta(\mathrm{ppm}): 8.21(\mathrm{~d}, J=3.42 \mathrm{~Hz}, 2 \mathrm{H}), 7.88(\mathrm{~s}, 2 \mathrm{H}), 7.32$ $(\mathrm{d}, J=3.67 \mathrm{~Hz}, 2 \mathrm{H}), 0.48(\mathrm{~s}, 18 \mathrm{H}) .{ }^{13} \mathrm{C} \mathrm{NMR}\left(400 \mathrm{MHz}, \mathrm{CDCl}_{3}\right)$ $\delta$ (ppm): 152.66, 154.07, 140.23, 136.26, 136.12, 135.99, 128.60, 128.40, 128.21, 125.80, -6.25. EI-MS $(\mathrm{m} / \mathrm{z}):[\mathrm{M}]^{+}$calculated for $\mathrm{C}_{20} \mathrm{H}_{24} \mathrm{~N}_{2} \mathrm{~S}_{3} \mathrm{Sn}_{2} \mathrm{Na}$, 650.9043; found, 650.9025. Anal. calculated for $\mathrm{C}_{20} \mathrm{H}_{24} \mathrm{~N}_{2} \mathrm{~S}_{3} \mathrm{Sn}_{2}$ : C, 38.37; H, 3.86; N, 4.47; S, 15.36; found: C, 38.62; H, 3.87; N, 4.50; S, 15.38.

5,6-Difluoro-4,7-bis(5-(trimethylstannyl)thiophene-2-yl)benzo$[\boldsymbol{c}][\mathbf{1}, 2,5]$ thiadiazole (M4). Under an inert argon atmosphere, 2,2,6,6-tetramethylpiperdine $\left(0.73 \mathrm{~cm}^{3}, 4.33 \mathrm{mmol}\right)$ was dissolved in anhydrous THF $\left(10 \mathrm{~cm}^{3}\right)$. The solution was cooled to $-78{ }^{\circ} \mathrm{C}$ and $n$-butyl lithium $\left(1.73 \mathrm{~cm}^{3}, 4.33 \mathrm{mmol}, 2.5 \mathrm{M}\right.$ solution in hexane) was added dropwise. The resulting solution was allowed to stir at $-78{ }^{\circ} \mathrm{C}$. The reaction was then warmed to room temperature and stirred for 10 minutes. The solution was then cooled to $-78{ }^{\circ} \mathrm{C}$ and 5,6-difluoro-4,7-di(thiophen-2yl)benzo $[c][1,2,5]$ thiadiazole $(0.56 \mathrm{~g}, 1.67 \mathrm{mmol})$ in THF $\left(15 \mathrm{~cm}^{3}\right)$ was added dropwise. Upon complete addition, the solution was stirred at $-78{ }^{\circ} \mathrm{C}$ for 1 hour. Trimethyltin chloride (8.32 $\mathrm{cm}^{3}, 8.33 \mathrm{mmol}, 1.0 \mathrm{M}$ solution in THF) was added dropwise at $-78{ }^{\circ} \mathrm{C}$. The reaction was warmed to room temperature and left to stir overnight. Upon completion, saturated brine was added to quench the reaction. The product was extracted with DCM $\left(5 \times 100 \mathrm{~cm}^{3}\right)$, washed with brine $(3 \times$ $\left.100 \mathrm{~cm}^{3}\right)$, dried $\left(\mathrm{MgSO}_{4}\right)$ and the solvent removed in vacuo. The crude material was recrystallised from ethanol to afford (M4) as orange powder $(0.274 \mathrm{~g}, 0.414 \mathrm{mmol}, 25 \%) .{ }^{1} \mathrm{H}$ NMR $(400 \mathrm{MHz}$, $\left.\mathrm{CDCl}_{3}\right) \delta(\mathrm{ppm}): 8.36(\mathrm{~d}, J=3.67 \mathrm{~Hz}, 2 \mathrm{H}), 7.37(\mathrm{~d}, J=3.42 \mathrm{~Hz}$, $2 \mathrm{H}), 0.49(\mathrm{~s}, 18 \mathrm{H}) .{ }^{13} \mathrm{C}$ NMR $\left(250 \mathrm{MHz}, \mathrm{CDCl}_{3}\right) \delta$ (ppm): 150.91, 150.71, 149.04, 148.33, 148.13, 142.61, 137.06, 135.52, $135.39,135.26,131.72,131.55,1.01,-6.29,-8.15 .{ }^{19} \mathrm{~F}$ NMR 
$\left(250 \mathrm{MHz}, \mathrm{CDCl}_{3}\right) \delta(\mathrm{ppm}):-127.95$. EI-MS $(\mathrm{m} / \mathrm{z}):[\mathrm{M}]^{+}$calculated for $\mathrm{C}_{20} \mathrm{H}_{22} \mathrm{~F}_{2} \mathrm{~N}_{2} \mathrm{~S}_{3} \mathrm{Sn}_{2} \mathrm{Na}_{2}$, 686.8855; found, 686.8879. Anal. calculated for $\mathrm{C}_{20} \mathrm{H}_{22} \mathrm{~N}_{2} \mathrm{~S}_{3} \mathrm{~F}_{2} \mathrm{Sn}_{2}$ : C, 36.29; H, 3.35; N, 5.74; S, 14.53; found: C, 36.84; H, 3.36; N, 4.20; S, 13.87 .

Poly $\left[N-9^{\prime}\right.$-pentacosanyl-2,7-carbazole-alt-5,5-( $4^{\prime}, 7^{\prime}$-di-2-thienyl5,6-difluoro-benzo[c][1,2,5]-thiadiazole)] (PCDTffBT). M2 (138 mg, $0.204 \mathrm{mmol}$ ), M4 (135 mg, $0.204 \mathrm{mmol}), \mathrm{Pd}(\mathrm{OAc})_{2}(3.30 \mathrm{mg}$, $14.8 \mu \mathrm{mol})$ and tri $(o$-tolyl $)$ phosphine $(9.00 \mathrm{mg}, 29.6 \mu \mathrm{mol})$ were added to a $100 \mathrm{~cm}^{3}$ round bottom flask. The flask was placed under an inert atmosphere of argon using standard Schlenk line techniques. Anhydrous toluene $\left(10 \mathrm{~cm}^{3}\right)$ was added, the system degassed and heated to $90{ }^{\circ} \mathrm{C}$ for 48 hours. Upon completion, the reaction was cooled to room temperature and 2-(tributylstannyl)thiophene $\left(0.30 \mathrm{~cm}^{3}, 0.945 \mathrm{mmol}\right)$ was added. The mixture was degassed and heated at $90{ }^{\circ} \mathrm{C}$ for 1.5 hours. The mixture was cooled to room temperature and 2-bromothiophene $\left(0.30 \mathrm{~cm}^{3}, 3.10 \mathrm{mmol}\right)$ was added, the system degassed and heated at $90{ }^{\circ} \mathrm{C}$ for 2 hours. The reaction was cooled to room temperature and poured into methanol and left to stir overnight. The mixture was filtered through a membrane and the solids were cleaned using Soxhlet extraction with solvents in the order; methanol, acetone, hexane and toluene. The toluene fraction was concentrated in vacuo and precipitated in methanol. The resulting mixture was stirred overnight and the polymers collect via filtration as a deep purple powder (107 mg, 62\%) GPC toluene fraction, $M_{\mathrm{n}}=7700 \mathrm{~g} \mathrm{~mol}^{-1}, M_{\mathrm{w}}=11900 \mathrm{~g} \mathrm{~mol}^{-1}, \mathrm{PDI}=1.55 .{ }^{1} \mathrm{H} \mathrm{NMR}$ $\left(500 \mathrm{MHz}, \mathrm{C}_{2} \mathrm{D}_{2} \mathrm{Cl}_{4}, 100{ }^{\circ} \mathrm{C}\right)\left(\delta_{\mathrm{H}} / \mathrm{ppm}\right) 8.37(\mathrm{~m}, 2 \mathrm{H}), 8.11(\mathrm{~m}, 2 \mathrm{H})$, $7.61(\mathrm{~m}, 2 \mathrm{H}), 7.56(\mathrm{~m}, 2 \mathrm{H}), 4.68(\mathrm{br}, 1 \mathrm{H}), 2.39$ (br, 2H), 2.09 (br, 2H) 1.39-1.04 (br, 40), $0.84(\mathrm{~m}, 6 \mathrm{H})$. Anal. calculated for $\mathrm{C}_{51} \mathrm{H}_{61} \mathrm{~F}_{2} \mathrm{~N}_{3} \mathrm{~S}_{3}$ : C, 72.03; H, 7.23; N, 4.94; S, 11.31; found: C, 71.16; $\mathrm{H}, 7.33$; N, 4.72; S, 10.47 .

Poly[ $N-9^{\prime}$-pentacosanyl-3,6-difluoro-2,7-carbazole-alt-5,5-( $\left(4^{\prime}, 7^{\prime}\right.$ di-2-thienyl-benzo[c][1,2,5]-thiadiazole)] (PCffDTBT). PCffDTBT was synthesized according to the procedure outlined in PCDTfBT. M1 (145 mg, $0.204 \mathrm{mmol}$ ), M3 (128 mg, $0.204 \mathrm{mmol}$ ), $\mathrm{Pd}(\mathrm{OAc})_{2}(3.30 \mathrm{mg}, 14.8 \mu \mathrm{mol})$ and tri $(o$-tolyl $)$ phosphine $(9.00 \mathrm{mg}$, $29.6 \mu \mathrm{mol})$ were added to a $100 \mathrm{~cm}^{3}$ round bottom flask. The flask was placed under an inert atmosphere of argon using standard Schlenk line techniques. Anhydrous toluene $\left(10 \mathrm{~cm}^{3}\right)$ was added, the system degassed and heated to $90{ }^{\circ} \mathrm{C}$ for 48 hours. The polymer was obtained as a deep purple solid (112 mg, 65\%) GPC toluene fraction, $M_{\mathrm{n}}=11400 \mathrm{~g} \mathrm{~mol}^{-1}, M_{\mathrm{w}}=20600 \mathrm{~g} \mathrm{~mol}^{-1}$, $\mathrm{PDI}=1.81 .{ }^{1} \mathrm{H}$ NMR $\left(500 \mathrm{MHz}, \mathrm{C}_{2} \mathrm{D}_{2} \mathrm{Cl}_{4}, 100{ }^{\circ} \mathrm{C}\right)\left(\delta_{\mathrm{H}} / \mathrm{ppm}\right) 8.21$ (br, 2H), 7.95 (br, 2H), $7.72(4 \mathrm{H}, \mathrm{br}), 7.63$ (br, 2H), 4.59 (br, 2H), 2.35 (br, 2H), 2.07 (br, 2H), 1.39-1.12 (br, 40H), $0.84(\mathrm{~m}, 6 \mathrm{H})$. Anal. calculated for $\mathrm{C}_{51} \mathrm{H}_{61} \mathrm{~F}_{2} \mathrm{~N}_{3} \mathrm{~S}_{3}$ : C, 72.03; H, 7.23; N, 4.94; S, 11.31; found: C, 70.03; H, 6.23; N, 5.36; S, 12.35.

Poly[ $N-9^{\prime}$-pentacosanyl-3,6-difluoro-2,7-carbazole-alt-5,5-( $\left(4^{\prime}, 7^{\prime}-\right.$ di-2-thienyl-5,6-difluoro-benzo[c][1,2,5]-thiadiazole)] (PCffDTffBT). PCffDTffBT was synthesized according to the procedure outlined in PCDTfBT. M1 (145 mg, $0.204 \mathrm{mmol}$ ), M4 (135 mg, $0.204 \mathrm{mmol}$ ), $\mathrm{Pd}(\mathrm{OAc})_{2}(3.30 \mathrm{mg}, 14.8 \mu \mathrm{mol})$ and tri(o-tolyl)phosphine $(9.00 \mathrm{mg}$, $29.6 \mu \mathrm{mol}$ ) were added to a $100 \mathrm{~cm}^{3}$ round bottom flask. The flask was placed under an inert atmosphere of argon using standard Schlenk line techniques. Anhydrous toluene $\left(10 \mathrm{~cm}^{3}\right)$ was added, the system degassed and heated to $90{ }^{\circ} \mathrm{C}$ for 48 hours. The polymer was obtained as a deep purple solid (40.8 mg, 23\%) GPC toluene fraction, $M_{\mathrm{n}}=6100 \mathrm{~g} \mathrm{~mol}{ }^{-1}, M_{\mathrm{w}}=8900 \mathrm{~g} \mathrm{~mol}^{-1}$, PDI $=1.46$. ${ }^{1} \mathrm{H}$ NMR $\left(500 \mathrm{MHz}, \mathrm{C}_{2} \mathrm{D}_{2} \mathrm{Cl}_{4}, 100{ }^{\circ} \mathrm{C}\right)\left(\delta_{\mathrm{H}} / \mathrm{ppm}\right) 8.38(\mathrm{~m}, 2 \mathrm{H}), 7.82-$ 7.59 (br, 6H), 4.60 (br, 1H), 2.34 (br, 2H), 2.07 (br, 2H), 1.39-1.03 (br, $40 \mathrm{H}), 0.83(\mathrm{~m}, 6 \mathrm{H})$. Anal. calculated for $\mathrm{C}_{51} \mathrm{H}_{59} \mathrm{~F}_{4} \mathrm{~N}_{3} \mathrm{~S}_{3}$ : C, 69.12; H, 6.71; N, 4.74; S, 10.85. Found: C, 63.88; H, 5.27; N, 5.26; S, 13.14.

\section{Acknowledgements}

We would like to thank the University of Sheffield for the award of a scholarship (L. C.) and EPSRC for financial support of this work via research grant EP/I028641/1.

\section{References}

1 Y. Cheng, S. Yang and C. Hsu, Chem. Rev., 2009, 109, 5868-5923.

2 V. N. Bliznyuk, S. A. Carter, J. C. Scott, G. Kla, R. D. Miller and D. C. Miller, Macromolecules, 1999, 32, 361-369.

3 W. Yue, Y. Zhao, S. Shao, H. Tian, Z. Xie and F. Wang, J. Mater. Chem., 2009, 19, 2199-2206.

4 A. Iraqi and I. Wataru, Chem. Mater., 2004, 42, 442-448.

5 K. Zhang, Y. Tao, C. Yang, H. You, Y. Zou, J. Qin and D. Ma, Chem. Mater., 2008, 20, 7324-7331.

6 N. Agarwal, P. K. Nayak, F. Ali, M. P. Patankar, K. L. Narasimhan and N. Periasamy, Synth. Met., 2011, 161, 466-473.

7 P. T. Boudreault, S. Wakim, L. Tang, Y. Tao, Z. Bao and M. Leclerc, J. Mater. Chem., 2009, 19, 2921-2928.

8 A. Van Dijken, J. J. A. M. Bastiaansen, N. M. M. Kiggen, B. M. W. Langeveld, C. Rothe, A. Monkman, I. Bach, P. Stössel and K. Brunner, J. Am. Chem. Soc., 2004, 126, 7718-7727.

9 B. N. Drolet, J. F. Morin, N. Leclerc, S. Wakim, Y. Tao and M. Leclerc, Adv. Funct. Mater., 2005, 15, 1671-1682.

10 Y. Sun, J. H. Seo, C. J. Takacs, J. Seifter and A. J. Heeger, Adv. Mater., 2011, 23, 1679-1683.

11 H. Yi, S. Al-Faifi, A. Iraqi, D. C. Watters, J. Kingsley and D. G. Lidzey, J. Mater. Chem., 2011, 21, 13649-13656.

12 Z. He, C. Zhong, X. Huang, W. Wong, H. Wu, L. Chen, S. Su and Y. Cao, Adv. Mater., 2011, 23, 4636-4643.

13 C. Gu, Y. Chen, Z. Zhang, S. Xue, S. Sun, C. Zhong, H. Zhang, Y. Lv, F. Li, F. Huang and Y. Ma, Adv. Energy Mater., 2014, 4, 1-5.

14 Y. Liu, J. Zhao, Z. Li, C. Mu, W. Ma, H. Hu, K. Jiang, H. Lin, H. Ade and H. Yan, Nat. Commun., 2014, 5, 1-8.

15 S. Beaupré and M. Leclerc, J. Mater. Chem. A, 2013, 1, 11097-11105.

16 J. W. Kingsley, P. P. Marchisio, H. Yi, A. Iraqi, C. J. Kinane, S. Langridge, R. L. Thompson, A. J. Cadby, A. J. Pearson, D. G. Lidzey, R. A. L. Jones and A. J. Parnell, Sci. Rep., 2014, 5286.

17 A. Casey, Y. Han, Z. Fei, A. J. P. White, T. D. Anthopoulos and M. Heeney, J. Mater. Chem. C, 2015, 3, 265-275. 
18 J. W. Jo, J. W. Jung, E. H. Jung, H. Ahn, T. J. Shin and W. H. Jo, Energy Environ. Sci., 2015, 8, 2427-2434.

19 G. Li, C. Kang, X. Gong, J. Zhang, W. Li, C. Li, H. Dong, W. Hu and Z. Bo, J. Mater. Chem. C, 2014, 2, 5116-5123.

20 N. Wang, Z. Chen, W. Wei and Z. Jiang, J. Am. Chem. Soc., 2013, 135, 17060-17068.

21 C. Du, W. Li, Y. Duan, C. Li, H. Dong, J. Zhu, W. Hu and Z. Bo, Polym. Chem., 2013, 4, 2773-2782.

22 J. Kim, M. H. Yun, G. Kim, J. Lee, S. M. Lee, S. Ko, Y. Kim, G. K. Dutta, M. Moon, S. Y. Park, D. S. Kim, J. Y. Kim and C. Yang, ACS Appl. Mater. Interfaces, 2014, 6, 7523-7534.

23 H. Wei, Y. Chao, C. Kang, C. Li, H. Lu, X. Gong, H. Dong, W. Hu, C. Hsu and Z. Bo, Macromol. Rapid Commun., 2015, 36, 84-89.

24 T. Umeyama, Y. Watanabe, E. Douvogianni and H. Imahori, J. Phys. Chem. C, 2013, 117, 21148-21157.

25 B. N. Blouin, A. Michaud and M. Leclerc, Adv. Mater., 2007, 19, 2295-2300.

26 L. Cartwright, A. Iraqi, Y. Zhang, T. Wang and D. G. Lidzey, RSC Adv., 2015, 5, 46386-46394.

27 Y. Zhang, S. C. Chien, K. S. Chen, H. L. Yip, Y. Sun, J. A. Davies, F. C. Chen and A. K. Y. Jen, Chem. Commun., 2011, 47, 11026-11028.

28 Z. Li, J. Lu, S.-C. Tse, J. Zhou, X. Du, Y. Tao and J. Ding, J. Mater. Chem., 2011, 21, 3226-3233.

29 S. Albrecht, S. Janietz, W. Schindler, J. Frisch, J. Kurpiers, J. Kniepert, S. Inal, P. Pingel, K. Fostiropoulos, N. Koch and D. Neher, J. Am. Chem. Soc., 2012, 134, 14932-14944.
30 H. Zhou, L. Yang, A. C. Stuart, S. C. Price, S. Liu and W. You, Angew. Chem., Int. Ed., 2011, 50, 2995-2998.

31 A. Iyer, J. Bjorgaard, T. Anderson and M. E. Köse, Macromolecules, 2012, 45, 6380-6389.

32 T. X. Carroll, T. D. Thomas, H. Bergersen, K. J. Børve and L. J. Sæthre, J. Org. Chem., 2006, 71, 1961-1968.

33 J. Rosenthal and D. I. Schuster, J. Chem. Educ., 2003, 80, 679-690.

34 R. Yamdagni and P. Kebarle, J. Am. Chem. Soc., 1976, 98, 1320-1324.

35 Z. B. Maksić, B. Kovačević and D. Kovaček, J. Phys. Chem. A, 1997, 101, 7446-7453.

36 H. Fang, J. W. Lin, I. H. Chiang, C. W. Chu, K. H. Wei and H. Lin, J. Polym. Sci., Part A: Polym. Chem., 2012, 50, 5011-5022.

37 H. Fukumoto and T. Yamamoto, J. Polym. Sci., Part A: Polym. Chem., 2008, 5, 2975-2982.

38 H. Li, P. Tang, Y. Zhao, S. X. Liu, Y. Aeschi, L. Deng, J. Braun, B. Zhao, Y. Liu, S. Tan, W. Meier and S. Decurtins, J. Polym. Sci., Part A: Polym. Chem., 2012, 50, 2935-2943.

39 T. Yamamoto, Q. Fang and T. Morikita, Macromolecules, 2003, 36, 4262-4267.

40 T. Yamamoto, Macromol. Rapid Commun., 2002, 23, 583-606.

41 G. Gritzner, Pure Appl. Chem., 1990, 62, 1839-1858.

42 H. Kun, H. Yi, R. G. Johnson and A. Iraqi, Polym. Adv. Technol., 2008, 19, 299-307. 